\title{
Structural Insights into UBAN-PolyUbs Assembly
}

\author{
Yu-Chih Lo ${ }^{\mathrm{a}}$, Jhen-Yi Hong ${ }^{\mathrm{a}}$, Su-Chang Lin ${ }^{\mathrm{b}}$ \\ ${ }^{a}$ Department of Biotechnology and Bioindustry Sciences, College of Bioscience and Biotechnology, \\ National Cheng Kung University, Tainan 70101, Taiwan, gracelo@mail.ncku.edu.tw \\ ${ }^{\mathrm{b}}$ Genomics Research Center, Academia Sinica, Taipei, Taiwan,
}

Recognition of linear polyubiquitin chains (polyUbs) by specific ubiquitin-binding proteins plays an important role in mediating NFkB signaling. The IKK complex is a master regulator in mediating NFKB activation, in which the regulatory subunit, NEMO, functions as a scaffold protein in binding with linear polyUbs assisting the IKK complex to be recruited to the TNFR-mediated signaling complexes (TNFRSCs) for activating downstream molecules. NEMO belongs to the UBAN family, a unique ubiquitin-binding protein family possessing the linear-ubiquitin-binding domain (UBD). Other UBAN family members including, A20-binding inhibitors of NFkB proteins (ABINs), and OPTN, however, attenuate NFkB signaling. Nevertheless, their recognition mechanisms for longer polyUbs are poorly understood, although the structural information for diUbs recognition has been extensively studied. Our recent structural studies provide a series of the crystal structures of the UBANs in complexes with different lengths of Ub chains, which represent the real images of how a ubiquitin-binding protein interacts with longer Ub chains. Together with the biochemical and biophysical assays, we further propose the molecular mechanism of how longer polyUbs recruit multiple signaling proteins to efficiently regulate signal transduction.

Reference

[1] SM Lin, SC Lin, JY Hong, TW Su, BJ Kuo, WH Chang, YF Tu, YC Lo*. Structural Insights into Linear TriUbiquitin Recognition by A20-Binding Inhibitor of NF- $\mathrm{KB}(\mathrm{ABIN})-2$. Structure. 25, p66-78. (2017) * corresponding author 